\title{
Sub-pathway analysis for severe burns injury patients: Identification of potential key IncRNAs by analyzing IncRNA-mRNA profile
}

\author{
GONGJIE TANG ${ }^{1}$, TAO ZHANG ${ }^{1}$, XINBO WANG $^{1}$, ZENGMEI SONG $^{1}$, \\ FUCUN LIU ${ }^{1}$, QIAN ZHANG ${ }^{1}$ and RAN HUO ${ }^{2}$ \\ ${ }^{1}$ Department of Burn and Plastic Surgery, Linyi Central Hospital, Linyi, Shandong 276400; \\ ${ }^{2}$ Department of Burn and Plastic Surgery, Shandong Provincial Hospital, Jinan, Shandong 250011, P.R. China
}

Received July 10, 2017; Accepted November 14, 2017

DOI: $10.3892 /$ etm.2018.6089

\begin{abstract}
The aim of the study was to identify key long non-coding RNAs (lncRNA) and related subpathways following severe burn injuries and research their functions. The miRNA-mRNA and IncRNA-miRNA interactions were downloaded from starBase v2.0 database. In addition, mRNA-miRNA interactions were obtained from TarBase, mirTarBase, mir2Disease, miRecords (V4.0) databases. The relationships of IncRNA-miRNA-mRNA were constructed. Genes of expression profiling were intersected with mRNA and IncRNA in lncRNA-mRNA interaction. Screened mRNAs were enriched into various pathways and screened lncRNAs were embedded into candidate pathways. Wallenius approximation methods were used to calculate the false discovery rate value of each sub-pathway. Based on the results of significant sub-pathways, the related lncRNA-mRNA network was constructed. A total of 18,081 genes were obtained. The lncRNA-mRNA intersections including 835 lncRNAs, 1,749 mRNAs and 7,693 interacting pairs were constructed. The enriched mRNAs were further enriched into various candidate pathways such as ribosome biogenesis in eukaryotes. Several sub-pathways were screened, including ribosome biogenesis in eukaryotes and MAPK signaling pathway. The network of pathway-lncRNA-mRNA was constructed. Hub-genes were identified, including C14orf169 and YLPM1. Several hub-lncRNAs were obtained, including PRKAG2 antisense RNA 1 and LEF1 antisense RNA 1. Several hub-lncRNAs including C14orf169, YLPM1, TTTY15, and PCBP1-AS1 were screened. The sub-pathways regulated by these lncRNAs were identified, and functions were predicted.
\end{abstract}

Correspondence to: Dr Ran Huo, Department of Burn and Plastic Surgery, Shandong Provincial Hospital, 324 Jingwu Road, Jinan, Shandong 250011, P.R. China

E-mail: tgj0539@126.com

Key words: subpathway analysis, severe burns injury, lncRNA, lncRNA-mRNA profile, bioinformatic analysis

\section{Introduction}

Burn injury is a common traumatic injury with considerable mortality and morbidity, requiring a long period of hospitalization and rehabilitation (1). Severe injury may induce a series of physiological changes, such as the change of metabolic rate, core temperature and various substrate cycling (2). In addition, both complications and burn wound of burn injury influence the physical and psychological health (3).

In previous studies, the expression of various genes was found to be altered after severe burns. Noronha et al (4) found that several genes, including $\mathrm{C}-\mathrm{X}-\mathrm{C}$ motif chemokine ligand 8 , interleukin 6 , tumor necrosis factor and major histocompatibility complex, Class I, E played a contributory role in wound infection induced by burns. In addition, Padfield et al identified various genes that encode chemokines, complement, oxidative-stress and immune functions following severe burn trauma (5). Long non-coding RNAs (lncRNA) were defined as non-protein coding transcripts longer than 200 nucleotides (6). Initially they were regarded as 'noise' with no biological function in the transcription of the genome. However, lncRNAs were confirmed to regulate gene expression in epigenetics, transcription and at the post-transcriptional level (7). In addition, lncRNAs were closely associated with the occurrence, development and prevention of disease by participating in chromatin modification, transcriptional activation, transcriptional interference and intranuclear transport (8). Haijun et al found a differential expression of lncRNAs compared to controls in skeletal muscles of rats with burn injuries (9). However, lncRNA and related functions and pathways in human have not been studied.

Identification of sub-pathways regulated by lncRNA are essential for monitoring of the occurrence and development of disease, and also help to research the functions of lncRNAs. In this study, we suggested a new method for identification of function regulated by lncRNA. The expression profiles of lncRNA and mRNA were integrated. Moreover, the regulation role of miRNA-target mRNA and topological analysis of pathways were processed to identify sub-pathways regulated 
A

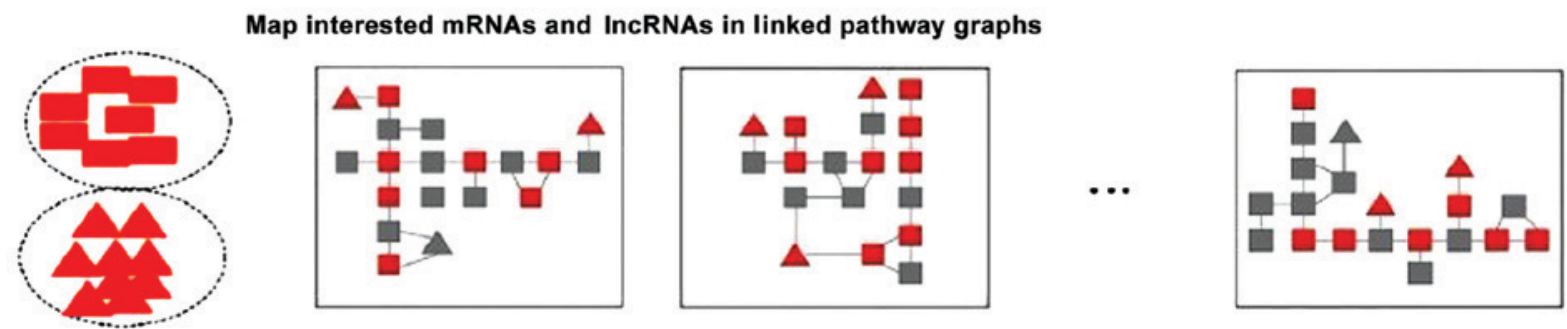

B

Identifing IncRNA-regulated sub-pathways
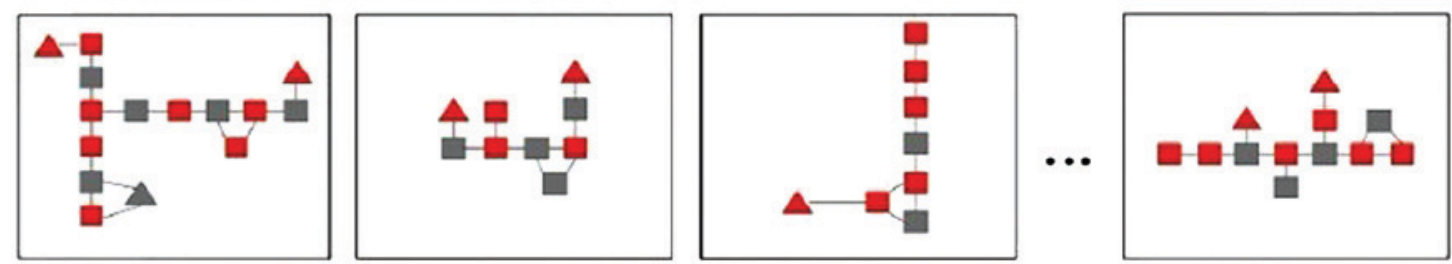

Figure 1. Analysis process of identification of IncRNA-regulated sub-pathways. LncRNA, long non-coding RNAs.

by lncRNA and predict the function of lncRNAs in severe burn injury patients.

\section{Materials and methods}

Samples. The expression profiling of E-GEOD-37069 with 553 injury samples and 37 controls, were downloaded from ArrayExpress Archive (http://www.ebi.ac.uk/arrayexpress/), which was based on the platform of [HG-U133_Plus_2] Affymetrix Human Genome U133 Plus 2.0 Array (Affymetrix, Santa Clara, CA,USA). These 553 injury samples were gathered from 244 severe burns patients over time. The patients had burns covering $>20 \%$ of the total surface and were admitted at $96 \mathrm{~h}$. The raw profiles were downloaded for further analysis.

Data preprocessing and genes screening. Based on the database of hgu133plus2, the raw data were preprocessed. Genes were obtained by combing various corresponding probes, and the expression value of this gene was assigned by the mean value of these probes.

Construction of candidate lncRNA-mRNA interaction. miRNA-mRNA interactions and lncRNA-miRNA interactions were downloaded from starBase v2.0 database. Moreover, some mRNA-miRNA interactions were obtained from various databases including TarBase, mirTarBase, mir2Disease, and miRecords (V4.0). According to shared miRNA in the aforementioned interactions, the relationships of lncRNA-miRNA-mRNA were constructed, and candidate lncRNA-mRNA regulatory relationships were obtained. The IncRNA-mRNA regulatory relationships were screened using the criteria: i) Hypergeometric functions were used to assess the significance of miRNA that have a regulatory relationship with both lncRNA and mRNA. The Benjamini and Hochberg (BH) method was used to adjust the P-value. The lncRNA-miRNA intersections with false discovery rate (FDR) $<0.05$ were screened. ii) Top 20\% IncRNA-mRNA intersections with higher Jaccard coefficient were retained. The formula of hypergeometric functions was shown as:

$$
\mathrm{P}=1-\sum_{t=0}^{x} \frac{\left(\begin{array}{l}
K \\
t
\end{array}\right)\left(\begin{array}{l}
N-K \\
M-t
\end{array}\right)}{\left(\begin{array}{l}
N \\
M
\end{array}\right)}
$$

Construction of co-expression lncGenePairs. The genes of expression profiling were intersected with mRNA and lncRNA in a lncRNA-mRNA interaction, respectively. Based on these matched expression profiles of IncRNA and mRNA, the Pearson's correlation coefficient was used to assess candidate lncRNA-mRNA intersections (10), and Fisher Z transformation was processed to calculate the P-value of lncRNA-mRNA-intersections (11). Furthermore, the P-value was adjusted as per the FDR using the $\mathrm{BH}$ method, and IncRNA-mRNA-intersections with FDR $<0.05$ were screened with significance (12).

Sub-pathway analysis. The lncRNA-regulated sub-pathway comprised two steps: Mapping interested mRNAs and IncRNAs into linked pathways, and identifying lncRNA-regulated sub-pathways (Fig. 1).

Pathway enrichment analysis of screened $m R N A$. Based on the Kyoto Encyclopedia of Genes and Genomes database, the screened mRNAs were enriched into various pathways. Fisher's exact test was used to identify significant pathways. Then, the P-value was adjusted into FDR by the BH method. The pathways with FDR $<0.01$ were regarded as candidate different pathways.

LncRNAs embed into enriched pathways. The screened IncRNAs were embedded into candidate pathways according to the results of lncGenePairs. Then, these pathways enriched lncRNA and related genes were obtained. 
Table I. The top 10 hub-lncRNA genes.

\begin{tabular}{llcr}
\hline Lnc & Gene & corValue & P-value \\
\hline EMX2OS & ACTN3 & $4.23 \times 10^{-1}$ & $4.80 \times 10^{27}$ \\
TBX5-AS1 & ADAMTS16 & $3.47 \times 10^{-1}$ & $3.79 \times 10^{18}$ \\
IQCH-AS1 & ANKRD37 & $9.48 \times 10^{-2}$ & $2.12 \times 10^{-2}$ \\
MFI2-AS1 & APLP1 & $4.20 \times 10^{-1}$ & $1.16 \times 10^{26}$ \\
HOXA-AS2 & ARL16 & $8.5 \times 10^{-2}$ & $3.8 \times 10^{-2}$ \\
HOXA-AS2 & ASB9 & $9.97 \times 10^{-2}$ & $1.53 \times 10^{-2}$ \\
FOXN3-AS1 & ASPSCR1 & $3.31 \times 10^{-1}$ & $1.22 \times 10^{16}$ \\
LBX2-AS1 & ASTN1 & $1.77 \times 10^{-1}$ & $1.43 \times 10^{05}$ \\
LEF1-AS1 & BBIP1 & $2.97 \times 10^{-2}$ & $1.71 \times 10^{13}$ \\
TDRG1 & BNC1 & $2.55 \times 10^{-2}$ & $6 \times 10^{10}$ \\
\hline
\end{tabular}

Table II. The top 5 pathways enriched by candidate mRNAs.

\begin{tabular}{lcc}
\hline Pathway_id & $\begin{array}{c}\text { Pathway_ } \\
\text { P-value }\end{array}$ & $\begin{array}{c}\text { P.adjust } \\
\text { (FDR) }\end{array}$ \\
\hline $\begin{array}{l}\text { hsa03008: Ribosome } \\
\text { biogenesis in eukaryotes }\end{array}$ & $8.19 \times 10^{5}$ & $8.19 \times 10^{5}$ \\
$\begin{array}{l}\text { hsa04722: Neurotrophin } \\
\text { signaling pathway }\end{array}$ & $8.07 \times 10^{5}$ & $8.07 \times 10^{5}$ \\
$\begin{array}{l}\text { hsa05216: Thyroid cancer } \\
\text { hsa05222: Small cell }\end{array}$ & $7.99 \times 10^{6}$ & $7.99 \times 10^{6}$ \\
$\begin{array}{l}\text { lung cancer } \\
\text { hsa05145: Toxoplasmosis }\end{array}$ & $7.59 \times 10^{6}$ & $7.59 \times 10^{6}$ \\
\hline
\end{tabular}

FDR, false discovery rate.

Identification of sub-pathways regulated by IncRNA. The screened lncRNA and related mRNA were regarded as flag nodes. Based on the situation of IncRNA embedding, the shortest route between two flag nodes was calculated. Once the molecular number between two flag nodes $\geq 1$, the two flag nodes were combined to one node. In addition, the nodes in pathways were also calculated, and pathways with $\geq 8$ nodes were regarded as candidate sub-pathways.

Assessment of sub-pathways and identification of hub-lncRNA. The Wallenius approximation methods were used to calculate the FDR value of each sub-pathway. In this process, the pathway weight was calculated using the formula:

$$
\mathrm{W}=1+\beta\left(-\log _{2}\left(\frac{G_{L}}{P_{G}}\right)\right)
$$

While the significance was evaluated using the formula of P-value $=\mathrm{F}\left(\mathrm{x}, \mathrm{m} 1, \mathrm{~m} 2\right.$, $\mathrm{n}$, weight $\left.\mathrm{i}_{\mathrm{i}}\right)$, where $\mathrm{x}$, represents the number of IncGenePairs that were enriched into this hub-pathway; $\mathrm{n}$, was the total number of mRNA; $\mathrm{m} 1$, represents the number of mRNA enriched into this hub-pathway; $\mathrm{m} 2$, the number of mRNA that could be annotated to lncGenePairs; $\mathrm{w}$, was the weight of this hub-subway; P and G were mRNA numbers in this sub-pathway; $\mathrm{G}$ and $\mathrm{L}$ were mRNA numbers regulated by lncRNA; $\beta=1$. In addition, the sub-pathway with FDR $<0.01$ was screened. Based on the results of significant sub-pathways, the related lncRNA-mRNA network was constructed. The lncRNAs with a degree more than the average degree were regarded as hub-lncRNAs.

\section{Results}

Genes screening and obtaining of lncRNA-mRNA interaction. After data preprocessing and combination, 18,081 genes were obtained. In addition, IncRNA-mRNA intersections including 835 lncRNAs, 1,749 mRNAs and 7,693 interacting pairs were constructed by several databases and sharing miRNAs.

Construction of co-expression lncGenePairs. Taking intersection between screened genes and lncRNA-mRNA, 1,641 mRNA expression profiles and 125 lncRNA expression profiles were obtained. After Fisher Z transformation, 656 lncGenePairs including 101 lncRNAs, and 503 mRNAs were constructed with $\mathrm{P}<0.05$. The top $5 \operatorname{lncGenePairs}$ were EMX2 opposite strand/antisense RNA-ACTN3 $\left(\mathrm{P}=4.80 \times 10^{27}\right)$, TBX5-AS1ADAMTS16 $\left(\mathrm{P}=3.79 \times 10^{18}\right)$, IQCH-AS1-ANKRD37 ( $\left.\mathrm{P}=0.02\right)$, MFI2-AS1-APLP1 $\left(\mathrm{P}=1.16 \times 10^{26}\right)$ and HOXA-AS2-ARL16 $(\mathrm{P}=0.04)$ (Table I).

Sub-pathway analysis. The enriched mRNA were enriched into various candidate pathways including ribosome biogenesis in eukaryotes $\left(\mathrm{FDR}=8.19 \times 10^{5}\right)$, neurotrophin signaling pathway $\left(\right.$ FDR $\left.=8.07 \times 10^{5}\right)$ and thyroid cancer $\left(\right.$ FDR $\left.=7.99 \times 10^{6}\right)$ (Table II).

After identification and assessment, several sub-pathways were screened, including ribosome biogenesis in eukaryotes, and the MAPK and ErbB signaling pathways (Table III). In the top 3 pathways, various IncRNA and related genes were involved. For example, YLP motif containing 1 (YLPM1) in ribosome biogenesis pathway was closely associated with heat repeat containing 1 (HEATR1), WD repeat domain 3 and spermatogenesis associated 5 (Fig. 2). Besides, HOXA transcript antisense RNA, myeloid-specific 1 in MAPK signaling pathway was regulated by AKT serine/threonine kinase 3 (AKT3), ribosomal protein S6 kinase A5 and mitogen-activated protein kinase kinase 6 (Fig. 3). Furthermore, YLPMI in the ErbB signaling pathway was associated with MAP2K1 and AKT3 (Fig. 4).

Identification of hub-lncRNA. Based on the above results, the network of pathway-lncRNA-mRNA was constructed (Fig. 5). Through topological analyses of this network, the hub-genes were identified, including chromosome 14 open reading frame 169 (C14orf169), (YLPM1), testis-specific transcript, Y-linked 15 (TTTY15), PCBP1 antisense RNA 1 (PCBP1-AS1) and deleted in lymphocytic leukemia 2. More importantly, several hub-lncRNAs were obtained, including PRKAG2 antisense RNA 1, LEF1 antisense RNA 1, EMX2 opposite strand/antisense RNA (EMX2OS) and BOLA3 antisense RNA 1. 
Table III. The top 5 hub-pathways.

\begin{tabular}{|c|c|c|c|c|c|c|}
\hline $\begin{array}{l}\text { Pathway } \\
\text { _id }\end{array}$ & $\begin{array}{l}\text { Pathway } \\
\text { _name }\end{array}$ & $\begin{array}{l}\text { Molecule ratio } \\
\qquad(\mathrm{m} 2 / \mathrm{x})\end{array}$ & $\begin{array}{l}\text { BgRatio } \\
(\mathrm{m} 1 / \mathrm{n})\end{array}$ & Weight & P-value & FDR \\
\hline 03008_1 & $\begin{array}{l}\text { Ribosome biogenesis } \\
\text { in eukaryotes }\end{array}$ & $15 / 489$ & $19 / 26232$ & 1.341037 & $<0.001$ & 0 \\
\hline 04010_1 & MAPK signaling pathway & $38 / 489$ & $51 / 26232$ & 1.387023 & $<0.001$ & 0 \\
\hline 04012_1 & ErbB signaling pathway & $18 / 489$ & $25 / 26232$ & 1.556393 & $<0.001$ & 0 \\
\hline 04066_2 & HIF-1 signaling pathway & $20 / 489$ & $25 / 26232$ & 1.395929 & $<0.001$ & 0 \\
\hline 04110_1 & Cell cycle & $26 / 489$ & $35 / 26232$ & 1.428843 & $<0.001$ & 0 \\
\hline
\end{tabular}

FDR, false discovery rate.

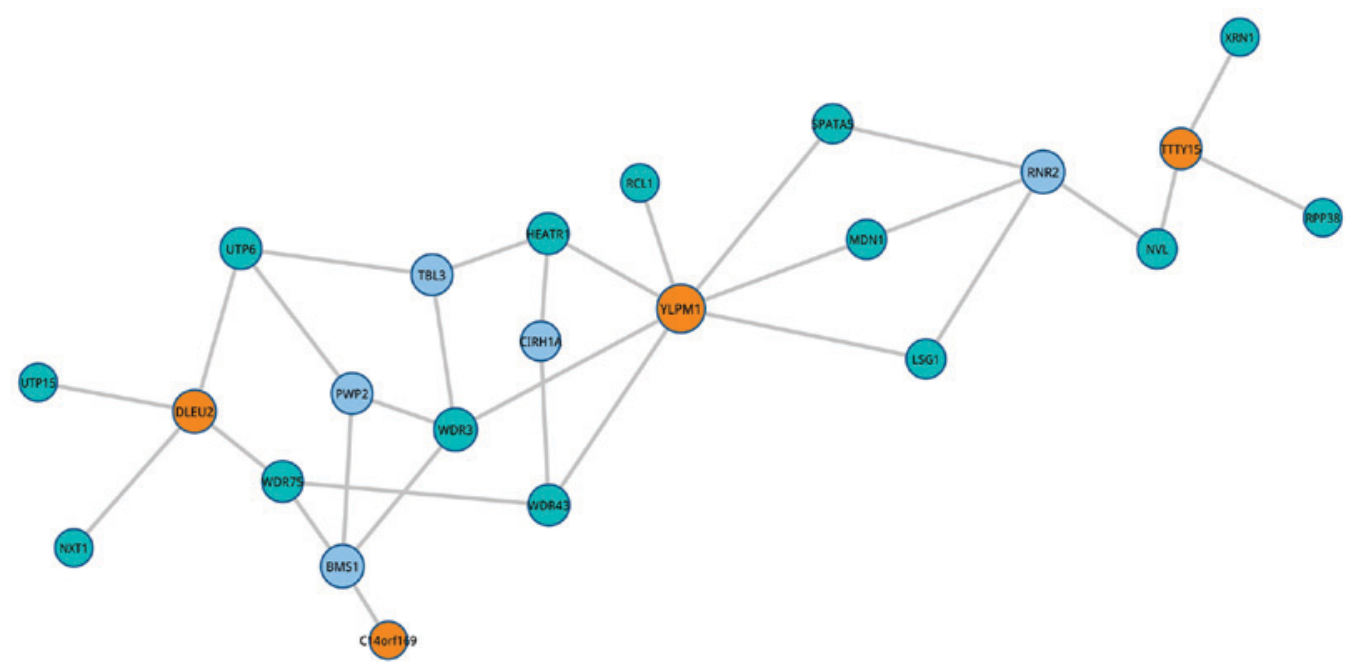

Figure 2. Pathway of ribosome biogenesis in eukaryotes related to lncRNAs and genes. The blue and yellow nodes represent lncRNAs and genes, respectively. The dark and light nodes represent nodes in and out of pathways, respectively. Edges represent regulated relationship between lncRNAs and genes. LncRNA, long non-coding RNAs.

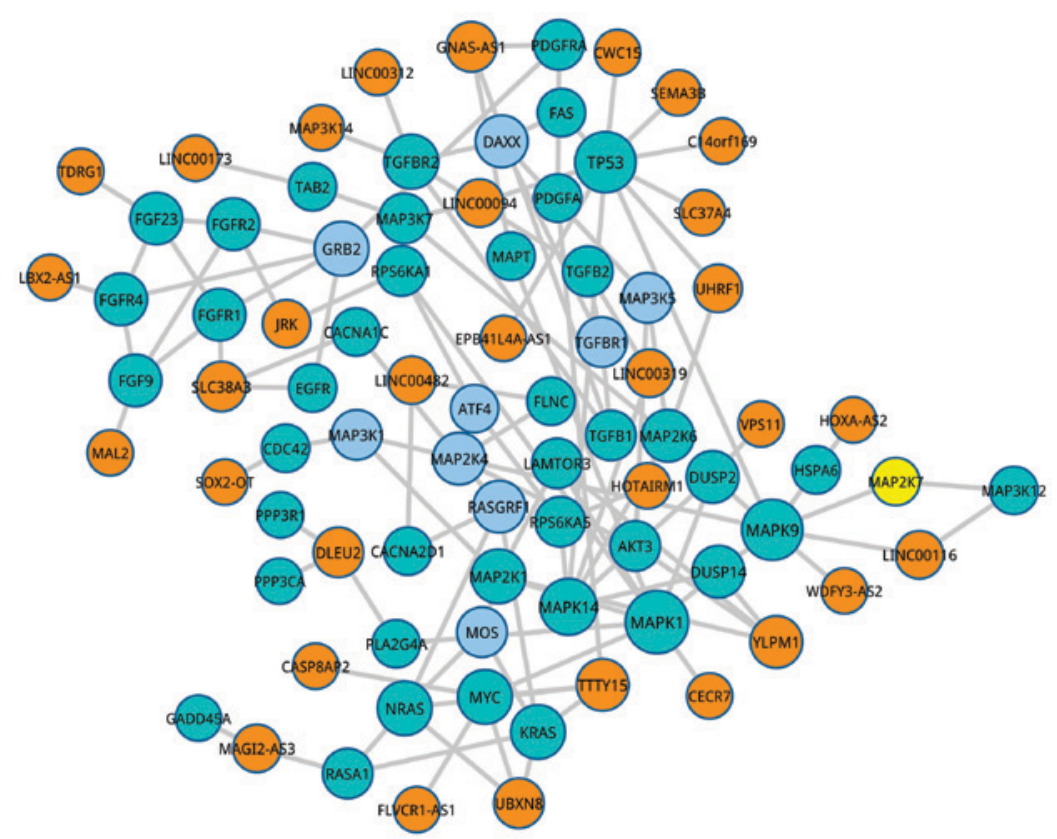

Figure 3. MAPK signaling pathway-related lncRNAs and genes. The blue and yellow nodes represent lncRNAs and genes, respectively. The dark and light nodes represent nodes in and out of pathways, respectively. Edges represent regulated relationship between lncRNAs and genes. LncRNA, long non-coding RNAs. 


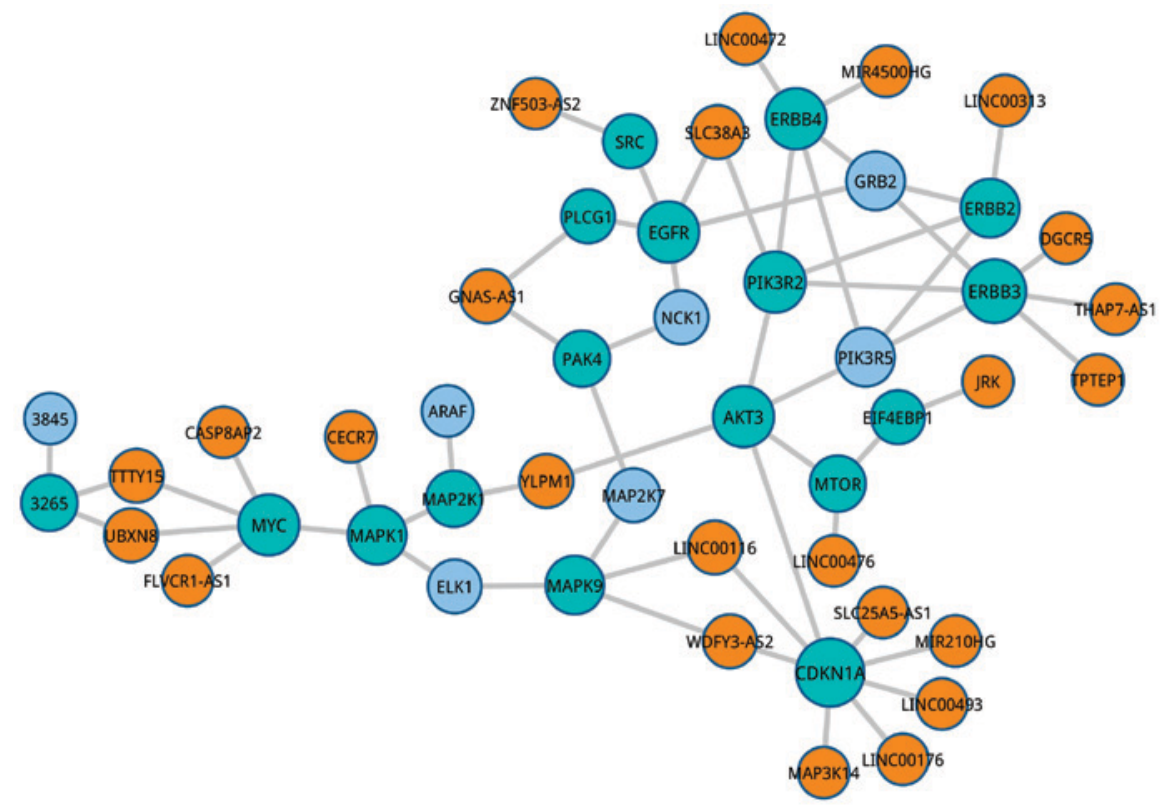

Figure 4. ErbB signaling pathway-related lncRNAs and genes. The blue and yellow nodes represent lncRNAs and genes, respectively. The dark and light nodes represent nodes in and out of pathways, respectively. Edges represent regulated relationship between lncRNAs and genes. LncRNA, long non-coding RNAs.

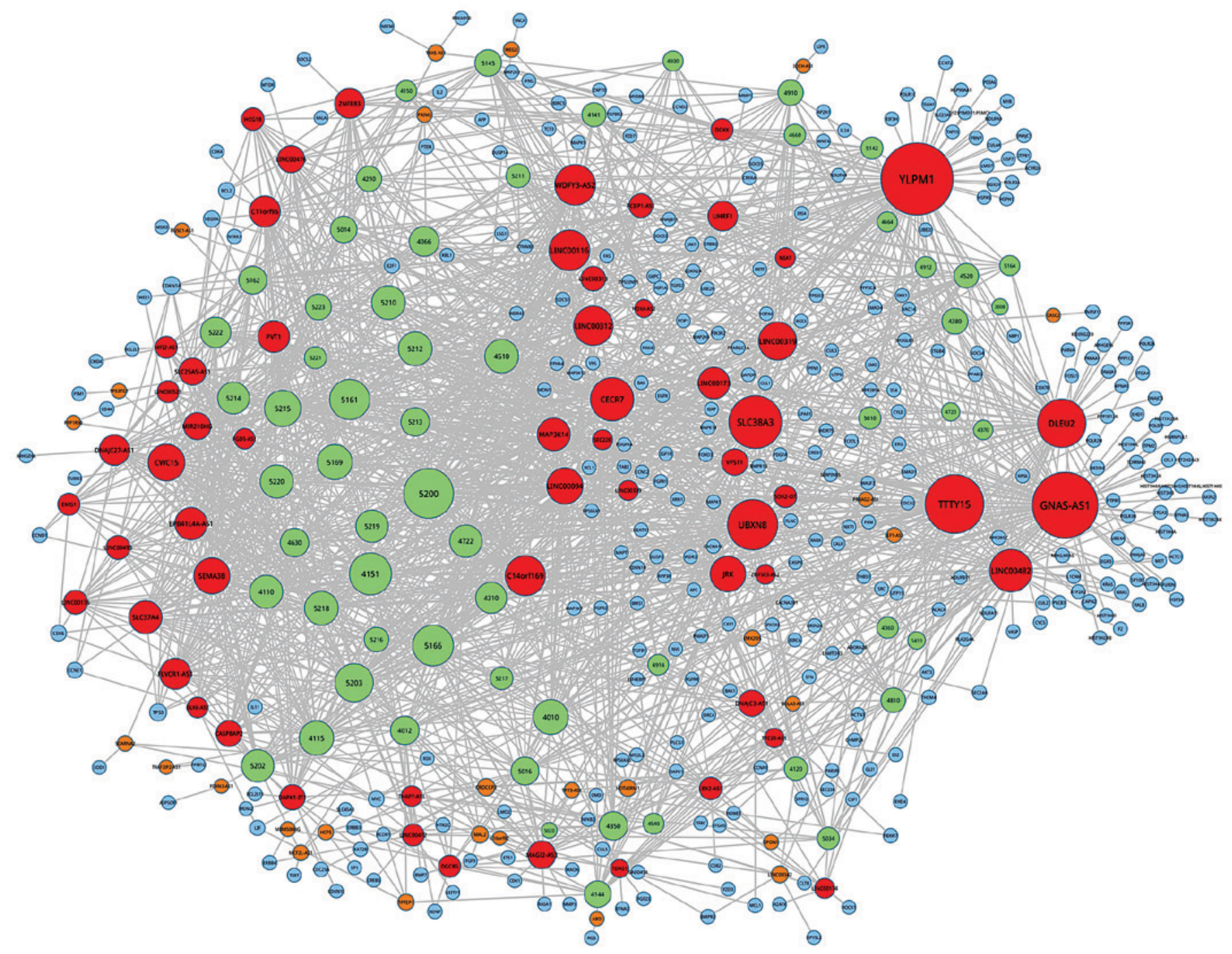

Figure 5. The network of pathway-lncRNA-mRNA. The red, yellow, light blue, dark blue and green nodes represent hublnc, lnc, mRNA, mRNA and path, respectively. The size of node represent degree of node. Edges represented regulated relationship among hublnc, lnc, mRNA, mRNA\& and path. 


\section{Discussion}

Increasing evidence has shown that lncRNAs were important factors for regulating gene expression (13). However, the molecular mechanism in burn injuries including lncRNA functions and regulatory genes remain unknown. In the present study, an effective method for identification of lncRNA-regulated functions was presented.LncRNA-regulated subpathways were identified, including ribosome biogenesis in eukaryotes, as well as MAPK and ErbB signaling pathways. More importantly, several hub-lncRNAs were identified, including C14orf169, YLPM1, TTTY15, and PCBP1-AS1. In addition, the sub-pathways regulated by the IncRNAs were identified, and functions of these IncRNAs were predicted.

C14orf169 was found to regulate various genes in this study, including eukaryotic translation initiation factor 1 (EIF1) and PPP2R5C. Thus EIF1 was mainly present in nucleus and cytosol. In a previous study, EIF1 was confirmed to be related to the generation of stable $40 \mathrm{~S}$ preinitiation complex (13). In addition, the eukaryotic translation initiation factor contributed to muscle regeneration by participating in the skeletal muscle stem cell differentiation (14). Besides, PPP2R5C was found to be overexpressed in myeloid leukemia cells, and closely related to leukemic cell proliferation and differentiation (15). In the present study, PPP2R5C was enriched into the PI3K-Akt and Wnt signaling pathways. Consistent with the study of Shi et al (16), anti-fibrotic effects by activation of the PIK3-Akt signaling pathway was beneficial for the reduction and prevention of scar formation. Furthermore, alteration of the Wnt signaling pathway could induce muscle fibrosis, and even lead to failure in muscle generation and tissue disorganization (17). Thus, C14orf169 served as a potential key lncRNA for the recovery of severe burn injury and post-operative complications by regulating EIF1 and PPP2R5C.

Moreover, YLPM1 was screened as a hub-lncRNA in the present study and resulted in the regulation of various genes, such as RPL10L, PPT1,HEATR1 and RPL9. Both RPL10L and $R P L 9$ encoded ribosomal proteins, and were mainly present in cytosol and nucleus. Kim et al (18) found that RPL9 interacted with Ftn-2, and played an important role in the maintenance of iron homeostasis. In addition, ribosomal protein phosphorylation was confirmed to interact with myostatin, and further affect the mature muscle (19). In mouce models, knockout PPT1 induced neuronal ceroid lipofuscinosis (20). In several diseases, HEATR1 was confirmed to regulate functional cytotoxic $\mathrm{T}$ lymphocytes and enhance cell immunity $(21,22)$. In the present study, HEATR1 was also found to enrich into the hub-pathway of ribosome biogenesis in eukaryotes. Based on this information, we inferred that YLPM1 was an important lncRNA after severe burn injuries by regulating RPL1OL, PPT1, HEATR1 and RPL9.

TTTY15 has been widely researched and found to have functions in the pathogenesis of prostate cancer, but it is less known regarding other diseases. In the present study, the gene was found to regulate the expression of different genes including KCTD13, UFL1 and ZNF322. In zebrafish models, KCTD13 was found to be closely associated with neurodevelopmental phenotype (23). In addition, KCTD13 interacted with Bacurd1, and further influenced the dendritic maturation and long-term positioning of cerebral neurons (24). It is well known that UFL1 is mainly involved in ufmylation of ubiquitin fold modifier 1 targets, which was necessary for erythroid differentiation $(25,26)$. Besides, ZNF322 is a crucial gene that regulates transcriptional activation in MAPK signaling pathways (27). Consequently, TTTY15 was important for recovery of severe burn injury by regulating KCTD13, UFL1 and ZNF322.

PCBP1-AS1, which was closer to the gene of PCBP1, resulted in the regulation of the expression of various genes including ZAP70, SOCS5 and SIRT1. Moreover, these regulated genes were involved in the T-cell receptor and Jak-STAT signaling pathways. In 2005, Wu et al (21) found that ZAP70 activated the generation of T-cell receptor microclusters and sustained T-cell activation, and participated in the immune response. Furthermore, SOCS5 was confirmed to be a specific inhibitor of IL-4 signaling, which was important for eosinophil infiltration (28). Of note, Schug et al (29) found that SIRT1 had a beneficial role in the treatment of inflammation and other associated diseases. Thus, PCBP1-AS1 may be involved in the mechanism following severe burn injury by regulating, the immune and inflammation process.

In conclusion, by integrating the expression profiles of lncRNA and mRNA, several hub-lncRNAs including C14orf169, YLPM1, TTTY15, and PCBP1-AS1 were screened. In addition, the sub-pathways regulated by these lncRNAs were identified, and functions of these IncRNAs were predicted.

\section{Competing interests}

The authors declare that they have no competing interests.

\section{References}

1. Brusselaers N, Monstrey S, Vogelaers D, Hoste E and Blot S: Severe burn injury in Europe: A systematic review of the incidence, etiology, morbidity, and mortality. Crit Care 14: R188, 2010

2. Wolfe RR, Herndon DN, Jahoor F, Miyoshi H and Wolfe M: Effect of severe burn injury on substrate cycling by glucose and fatty acids. N Engl J Med 317: 403-408, 1987.

3. Pavoni V, Gianesello L, Paparella L, Buoninsegni LT and Barboni E: Outcome predictors and quality of life of severe burn patients admitted to intensive care unit. Scand J Trauma Resusc Emerg Med 18: 24, 2010.

4. Noronha SA, Noronha SM, Lanziani LE, Ferreira LM and Gragnani A: Innate and adaptive immunity gene expression of human keratinocytes cultured of severe burn injury. Acta Cir Bras 29: 60-67, 2014.

5. Padfield KE, Zhang Q, Gopalan S, Tzika AA, Mindrinos MN, Tompkins RG and Rahme LG: Local and distant burn injury alter immuno-inflammatory gene expression in skeletal muscle. J Trauma 61: 280-292, 2006.

6. Pian C, Zhang G, Chen Z, Chen Y, Zhang J, Yang T and Zhang L: LncRNApred: Classification of long non-coding RNAs and protein-coding transcripts by the ensemble algorithm with a new hybrid feature. PLoS One 11: e0154567, 2016.

7. Vadaie $\mathrm{N}$ and Morris KV: Long antisense non-coding RNAs and the epigenetic regulation of gene expression. Biomol Concepts 4 : 411-415, 2013.

8. Wu Z, Liu X, Liu L, Deng H, Zhang J, Xu Q, Cen B and Ji A:Regulation of IncRNA expression.Cell Mol Biol Lett 19: 561-575, 2014.

9. Haijun Z, Yonghui Y and Jiake C: Expression signatures of lncRNAs in skeletal muscles at the early flow phase revealed by microarray in burned rats. Ulus Travma Acil Cerrahi Derg 22: 224-232, 2016

10. Pearson K and Galton F: Pearson product-moment correlation coefficient. Covariance, 2014. 
11. Bond CF Jr and Richardson K: Seeing the Fisher Z -transformation. Psychometrika 69: 291-303, 2004.

12. Thissen D, Steinberg L and Kuang D: Quick and easy implementation of the Benjamini-Hochberg procedure for controlling the false positive rate in multiple comparisons. J Educ Behav Stat 27: 77-83, 2002.

13. Huarte M: The emerging role of lncRNAs in cancer. Nat Med 21: $1253-1261,2015$

14. Luchessi AD, Cambiaghi TD, Hirabara SM, Lambertucci RH, Silveira LR, Baptista IL, Moriscot AS, Costa-Neto CM and Curi R: Involvement of eukaryotic translation initiation factor 5A (eIF5A) in skeletal muscle stem cell differentiation. J Cell Physiol 218: 480-489, 2009.

15. Zheng H, Chen Y, Chen S, Niu Y, Yang L, Li B, Lu Y, Geng S, $\mathrm{Du} \mathrm{X}$ and $\mathrm{Li}$ Y: Expression and distribution of PPP2R5C gene in leukemia Open Access. J Hematol Oncol 4: 21-26, 2011.

16. Shi J, Li J, Guan H, Cai W, Bai X, Fang X, Hu X, Wang Y, Wang H, Zheng Z, et al: Anti-fibrotic actions of interleukin-10 against hypertrophic scarring by activation of PI3K/AKT and STAT3 signaling pathways in scar-forming fibroblasts. PLoS One 9: e98228, 2014.

17. Cisternas P, Henriquez JP, Brandan E and Inestrosa NC: Wnt signaling in skeletal muscle dynamics: Myogenesis, neuromuscular synapse and fibrosis. Mol Neurobiol 49: 574-589, 2014.

18. Kim HS, Ahn CH, Park TS, Park HD, Koh KS, Ryoo ZY Park SC and Lee S: Gene expression profiling of a cold-shocked earthworm Eisenia andrei. Cryo Letters 33: 1-11, 2012.

19. Welle S, Burgess K and Mehta S: Stimulation of skeletal muscle myofibrillar protein synthesis, p70 S6 kinase phosphorylation, and ribosomal protein S6 phosphorylation by inhibition of myostatin in mature mice. Am J Physiol Endocrinol Metab 296: E567-E572, 2009.

20. Gupta P, Soyombo AA, Atashband A,Wisniewski KE, Shelton JM, Richardson JA, Hammer RE and Hofmann SL: Disruption of PPT1 or PPT2 causes neuronal ceroid lipofuscinosis in knockout mice. Proc Natl Acad Sci USA 98: 13566-13571, 2001.

21. Wu ZB, Qiu C, Zhang AL, Cai L, Lin SJ, Yao Y, Tang QS, Xu M, Hua W, Chu YW, et al: Glioma-associated antigen HEATR1 induces functional cytotoxic T lymphocytes in patients with glioma. J Immunol Res 2014: 131494, 2014.
22. Liu T, Fang Y, Zhang H, Deng M, Gao B, Niu N, Yu J, Lee S, Kim J, Qin B, et al: HEATR1 negatively regulates Akt to help sensitize pancreatic cancer cells to chemotherapy. Cancer Res 76: 572-581, 2016.

23. Golzio C1, Willer J, Talkowski ME, Oh EC, Taniguchi Y, Jacquemont S, Reymond A, Sun M, Sawa A, Gusella JF, et al: KCTD13 is a major driver of mirrored neuroanatomical phenotypes associated with the $16 \mathrm{p} 11.2 \mathrm{CNV}$. Nature 485: 363-367, 2012.

24. Gladwyn-Ng I, Huang L, Ngo L, Li SS, Qu Z, Vanyai HK, Cullen HD, Davis JM and Heng JI: Bacurd1/Kctd13 and Bacurd2/Tnfaip1 are interacting partners to Rnd proteins which influence the long-term positioning and dendritic maturation of cerebral cortical neurons. Neural Dev 11: 7, 2016.

25. Zhang Y, Zhang M, Wu J, Lei G and Li H: Transcriptional regulation of the Ufm1 conjugation system in response to disturbance of the endoplasmic reticulum homeostasis and inhibition of vesicle trafficking. PLoS One 7: e48587, 2012.

26. Tatsumi K, Yamamoto-Mukai H, Shimizu R, Waguri S, Sou YS, Sakamoto A, Taya C, Shitara H, Hara T, Chung CH, et al: The Ufm1-activating enzyme Uba5 is indispensable for erythroid differentiation in mice. Nat Commun 2: 181, 2011.

27. Li Y, Wang Y, Zhang C, Yuan W, Wang J, Zhu C, Chen L, Huang W, Zeng W, Wu X, et al: ZNF322, a novel human C2H2 Kruppel-like zinc-finger protein, regulates transcriptional activation in MAPK signaling pathways. Biochem Biophys Res Commun 325: 1383-1392, 2004.

28. Ozaki A, Seki Y, Fukushima A and Kubo M: The control of allergic conjunctivitis by suppressor of cytokine signaling (SOCS) 3 and SOCS5 in a murine model. J Immunol 175 : 5489-5497, 2005.

29. Schug TT, Xu Q, Gao H, Peres-da-Silva A, Draper DW, Fessler MB, Purushotham A and Li X: Myeloid deletion of SIRT1 induces inflammatory signaling in response to environmental stress. Mol Cell Biol 30: 4712-4721, 2010.

This work is licensed under a Creative Commons Attribution-NonCommercial-NoDerivatives 4.0 International (CC BY-NC-ND 4.0) License. 